\title{
Outcomes from colonoscopy following referral from New Zealand general practice: a retrospective analysis
}

Ross Lawrenson ${ }^{1 *}$, Sheena Moosa², Judy Warren², Ralph van Dalen², Lynne Chepulis , Tania Blackmore', Chunhuan Lao ${ }^{1}$, Christopher Mayo ${ }^{1}$, Jacquie Kidd ${ }^{3}$, Melissa Firth ${ }^{1}$, Tim Stokes ${ }^{4}$, Mark Elwood ${ }^{5}$, David Weller ${ }^{6}$ and Jon Emery ${ }^{7}$

\begin{abstract}
Background: New Zealand has high rates of colorectal cancer (CRC) but poor outcomes. Most patients with CRC are diagnosed following referral from general practice, where a general practitioner (GP) assesses symptoms according to national guidelines. All referred patients are then re-prioritised by the hospital system. The first objective of this study was to identify what proportion of patients referred by general practice to surgical/gastroenterology at Waikato District Health Board (DHB) had a colonoscopy. The second objective was to determine what proportion of these referrals have an underlying CRC and the factors associated with the likelihood of this diagnosis.
\end{abstract}

Methods: This study is a retrospective analysis of e-referral data for patients aged 30-70+ who were referred from 75 general practices to general surgery, gastroenterology or direct to colonoscopy at Waikato DHB, 01 January 2015-31 December 2017. Primary and secondary outcome measures included the proportion and characteristics of patients who were having colonoscopy, and of those, who were diagnosed with CRC. Data were analysed using chi square and logistic regression.

Results: $6718 / 20648$ (32.5\%) patients had a colonoscopy and 372 (5.5\%) of these were diagnosed with CRC. The probability of having CRC following a colonoscopy increased with age ( $p$ value $<0.001)$. Females ( $p$ value $<0.001$ ), non-Māori ( $p$ value $<0.001$ ), and patients with a high suspicion of cancer (HSCan) label originating from their GP were more likely to have a colonoscopy, while the odds ratio of Māori having a colonoscopy was 0.66 ( $95 \% \mathrm{Cl} 0.60-0.73)$. The odds ratio of a CRC diagnosis following colonoscopy was 1.67 ( $95 \% \mathrm{Cl} 1.35-2.07$ ) for men compared to women, and 2.34 (95\% Cl 1.70-3.22) for those with a GP HSCan label. Of the 585 patients referred with a GP HSCan, 423 (72.3\%) were reprioritised by the hospital and 55 patients had their diagnosis unnecessarily delayed.

Conclusions: If a GP refers a patient with an HSCan, and the patient receives a colonoscopy, then the likelihood of having CRC is almost $15.0 \%$. This would suggest that these patients should be routinely prioritised without further triage by the hospital. Further research is needed to understand why Māori are less likely to receive a colonoscopy following referral from general practice.

Keywords: Colorectal cancer, Colonoscopy rates, General practice, High suspicion of cancer

*Correspondence: ross.lawrenson@waikato.ac.nz

${ }^{1}$ Medical Research Centre, University of Waikato, Hamilton, New Zealand Full list of author information is available at the end of the article

\section{Background}

Each year, approximately 1200 people in New Zealand (NZ) die of colorectal cancer (CRC) [1]. CRC occurs less frequently in Māori-the indigenous peoples of NZ prior 
to colonisation-compared to non-Māori [2]. Age, male gender, a family history and a raised body mass index (BMI) are recognised risk factors for CRC [3]. A personal history of adenomatous polyps or inflammatory bowel disease also increases risk [4]. Most patients with CRC are diagnosed following referral to a public hospital from a general practitioner (GP) $[5,6]$, who are asked to follow NZ Ministry of Health $(\mathrm{MOH})$ guidelines for specialist referral of patients with signs and symptoms of bowel cancer. These guidelines outline symptoms that can include: rectal bleeding, blood mixed with stool; change in bowel habit (for at least 6 weeks); abdominal pain or bloating; weight loss and anaemia [7]. Patients with these symptoms usually present to their GP who will then arrange investigations and referral to specialist services [8]. More recently, direct access referral has been made available for GPs to refer patients for colonoscopyagain within strict guidelines outlining symptoms such as unexplained rectal bleeding with iron deficiency anaemia, changed bowel habit, family history or known/suspected CRC [9]. In certain defined circumstances such as persistent rectal bleeding or a change in bowel habit GPs can indicate a High Suspicion of Cancer (HSCan), and under current NZ guidelines these patients should be seen urgently within 2 weeks. However, under the NZ HSCan guidelines these referrals would still be triaged by hospital specialist services who make the final decision as to whether the referral is deemed high suspicion or can be considered for semi-urgent or routine follow up.

Referral from general practice for a diagnosis of suspected bowel cancer and colonoscopy is a relatively rare event. With 3100 new cases of CRC each year [1], on average the $3700 \mathrm{NZ}$ GPs will see less than one new case of CRC per year-consistent with United Kingdom (UK) figures [10]. A Dutch study of 140,000 patients suggested only $2.0 \%$ of patients were referred for investigation of suspected CRC in a 5 year period [11]. What has not been widely reported is what proportion of patients referred for colonoscopy have an underlying cancer. Prior to the recent inauguration of a nationwide bowel cancer screening programme for $60-74$ year olds, a screening pilot [12] that involved 50-74 year olds, carried out by Waitematā District Health Board (DHB) reported 212 new cancers found after 4500 colonoscopies following a positive Faecal Immunochemical test (FIT) result, or $4.7 \%$. In a small study of 144 symptomatic patients with constipation from South Africa, it was found that 9/144 (6.25\%) had an underlying CRC [13]. In Koning's [11] general practice study only $2.0 \%(57 / 2785)$ of the patients who had a colonoscopy were diagnosed with CRC.

The Waikato DHB has a population of 430,000 and is located in the North Island of NZ. Twenty three percent of the Waikato population identify as Māori. The majority of cancer patients are managed in the public hospital system which is free to all NZ residents, but a small proportion of CRC patients may be seen and managed in the private health system. This study only relates to patients referred to the public health system. While generally all referrals are reviewed to see whether they will be offered as First Specialist Assessment (FSA), since 2016, for patients who have clear cut symptoms and are in the appropriate age range ( $>50$ years), GPs in the region have been able to make a direct referral for colonoscopy. However, these patients also require the approval of a specialist before a colonoscopy is arranged. Waikato DHB covers 75 general practices and it has been noted that the referral rates from practices vary greatly. It has been postulated that there is a correlation between referral rates and the risk of underlying pathology e.g., high referrers may have a lower positivity rate. It has been noted in the UK that using routine data on detection and conversion rates of different GPs should be interpreted with caution and is altered by the case mix of patients presenting [14]. The aim of our study is to identify the characteristics of patients aged 30-70+ referred to general surgical and gastroenterology outpatients (including direct referral) receiving a colonoscopy. We also wanted to establish risk of underlying CRC in those who had a colonoscopy and the factors associated with the likelihood of this diagnosis.

\section{Methods}

The population investigated were patients aged 30-70+ referred from 75 general practices to general surgery, gastroenterology or direct to colonoscopy at Waikato DHB from 01 January 2015 to 31 December 2017. Data were sourced from Waikato DHBs electronic referral system, where referrals are generated from general practice following assessment of patient symptoms according to $\mathrm{MOH}$ referral guidelines. The extracted dataset included patient age, gender, ethnicity, date of referral, whether the patient had colonoscopy, whether it was direct access colonoscopy, whether the general practice was a high referrer (defined as a referral rate above the median referral rate), GP label of HSCan, and the hospital triage label of HSCan (i.e., specialist assessment of risk based on information supplied by the GP on the e-referral form. This risk assessment of all patients is undertaken by the hospital irrespective of the GP recommendation). This dataset was then linked to the National Cancer Register through the National Health Index (NHI) number (a unique identifier for all who use health and disability services in NZ) to identify any cancer diagnosis for the referred patients from 01 January 2015 to 31 December 2018. Ethical approval was obtained from the Health and 
Disability Ethic Committee of New Zealand (Approval Number: 17/NTB/156).

The characteristics of patients who received a colonoscopy were analysed and compared to the characteristics of patients who did not receive a colonoscopy. The difference was examined with a Chi-square test. Logistic regression was used to estimate the adjusted odds ratio (OR) and the 95\% confidence interval (CI) of the odds ratio for these factors in the likelihood of colonoscopy.

We then analysed which patients were diagnosed with CRC following a colonoscopy. The characteristics of patients who had CRC were compared to patients who did not have CRC. Logistic regression was used to estimate the adjusted odds ratio for these factors in the likelihood of a CRC diagnosis. Cancer extent was described by colon cancer and rectal cancer. All data analyses were performed in IBM SPSS statistics 25 (New York, United States).

\section{Results}

During the study period, 20,648 patients were referred from 75 general practices to general surgery, gastroenterology or direct to colonoscopy. Of these, 6718 patients had a colonoscopy (Table 1 ). The probability of having a colonoscopy increased with age $(p$ value $<0.001)$. Female patients were slightly more likely to have a colonoscopy than male patients $(33.6 \%$ vs $31.2 \%, p$ value $<0.001)$, and non-Māori patients were more likely to have a colonoscopy than Mãori patients (33.9\% vs $23.7 \%, p$ value $<0.001)$. Patients with a GP label of HSCan or hospital label of HSCan were more likely to have a colonoscopy than those without an HSCan label.

As shown in Table 2, after adjustment for the factors shown, the odds ratio of Mãori patients having a colonoscopy was 0.66 (95\% CI 0.60-0.73). The adjusted odds ratio of the GP practice being a high referrer (i.e., above the median the median referral rate) in having a colonoscopy was 0.94 (95\% CI 0.88-1.00). The adjusted odds ratio of a GP label of HSCan and hospital label of HSCan in having a colonoscopy was 2.22 (95\% CI 1.92-2.56) and 1.74 (95\% CI 1.26-2.42), respectively. After adjustment, gender and year of referral did not have a significant impact on having a colonoscopy.

Among the patients who had a colonoscopy, 372 (5.5\%) were diagnosed with CRC (Table 3). The probability of having $\mathrm{CRC}$ increased with age, from $1.5 \%$ of patients

Table 1 Characteristics of patients referred

\begin{tabular}{|c|c|c|c|c|c|c|}
\hline \multirow{2}{*}{$\begin{array}{l}\text { Characteristics } \\
\text { Age group }\end{array}$} & \multicolumn{2}{|c|}{ No colonoscopy } & \multicolumn{2}{|c|}{ Had colonoscopy } & \multirow[t]{2}{*}{$p$ value } & \multirow[t]{2}{*}{ Overall } \\
\hline & & & & & & \\
\hline $30-49$ & 4415 & $78.0 \%$ & 1244 & $22.0 \%$ & $<0.001$ & 5659 \\
\hline $50-59$ & 2829 & $67.2 \%$ & 1381 & $32.8 \%$ & & 4210 \\
\hline $60-69$ & 2829 & $60.6 \%$ & 1843 & $39.4 \%$ & & 4672 \\
\hline $70+$ & 3857 & $63.2 \%$ & 2250 & $36.8 \%$ & & 6107 \\
\hline \multicolumn{7}{|l|}{ Gender } \\
\hline Female & 7483 & $66.4 \%$ & 3790 & $33.6 \%$ & $<0.001$ & 11,273 \\
\hline Male & 6447 & $68.8 \%$ & 2928 & $31.2 \%$ & & 9375 \\
\hline \multicolumn{7}{|l|}{ Ethnicity } \\
\hline Non-Māori & 11,759 & $66.1 \%$ & 6044 & $33.9 \%$ & $<0.001$ & 17,803 \\
\hline Māori & 2171 & $76.3 \%$ & 674 & $23.7 \%$ & & 2845 \\
\hline \multicolumn{7}{|l|}{ Year } \\
\hline 2015 & 4936 & $68.1 \%$ & 2315 & $31.9 \%$ & 0.250 & 7251 \\
\hline 2016 & 4488 & $66.8 \%$ & 2235 & $33.2 \%$ & & 6723 \\
\hline 2017 & 4506 & $67.5 \%$ & 2168 & $32.5 \%$ & & 6674 \\
\hline \multicolumn{7}{|l|}{ High referrer } \\
\hline Low & 4709 & $67.0 \%$ & 2321 & $33.0 \%$ & 0.290 & 7030 \\
\hline High & 9221 & $67.7 \%$ & 4397 & $32.3 \%$ & & 13,618 \\
\hline \multicolumn{7}{|l|}{ HSCan-GP } \\
\hline Yes & 522 & $47.2 \%$ & 585 & $52.8 \% \%$ & $<0.001$ & 1107 \\
\hline No & 13,408 & $68.6 \%$ & 6133 & $31.4 \%$ & & 19,541 \\
\hline \multicolumn{7}{|l|}{ HSCan-hospital } \\
\hline Yes & 221 & $48.8 \%$ & 232 & $51.2 \%$ & $<0.001$ & 453 \\
\hline No & 13,709 & $67.9 \%$ & 6486 & $32.1 \%$ & & 20,195 \\
\hline Overall & 13,930 & $67.5 \%$ & 6718 & $32.5 \%$ & & 20,648 \\
\hline
\end{tabular}


Table 2 Adjusted odds ratio of having a colonoscopy

\begin{tabular}{|c|c|c|c|}
\hline Factors & $p$ value & Odds ratio & $\begin{array}{l}95 \% \\
\text { confidence } \\
\text { interval }\end{array}$ \\
\hline \multicolumn{4}{|l|}{ Age } \\
\hline (continuous) & $<0.001$ & 1.01 & $(1.01-1.02)$ \\
\hline \multicolumn{4}{|l|}{ Gender } \\
\hline Female & Ref & & \\
\hline Male & $<0.001$ & 0.87 & $(0.82-0.93)$ \\
\hline \multicolumn{4}{|l|}{ Year } \\
\hline (continuous) & 0.707 & 0.99 & $(0.96-1.03)$ \\
\hline \multicolumn{4}{|l|}{ Ethnicity } \\
\hline Non-Māori & Ref & & \\
\hline Māori & $<0.001$ & 0.66 & $(0.60-0.73)$ \\
\hline \multicolumn{4}{|l|}{ High referrer } \\
\hline Low & Ref & & \\
\hline High & 0.048 & 0.94 & $(0.88-1.00)$ \\
\hline \multicolumn{4}{|l|}{ HSCan-GP } \\
\hline No & Ref & & \\
\hline Yes & $<0.001$ & 2.22 & $(1.92-2.56)$ \\
\hline \multicolumn{4}{|l|}{ HSCan-hospital } \\
\hline No & Ref & & \\
\hline Yes & $<0.001$ & 1.74 & $(1.26-2.42)$ \\
\hline \multicolumn{4}{|l|}{ Interaction term } \\
\hline $\begin{array}{l}\text { (HSCan- } \\
\text { GP } \times \text { HSCan- } \\
\text { Hospital) }\end{array}$ & 0.009 & 0.57 & $(0.37-0.87)$ \\
\hline
\end{tabular}

aged $30-49$ years to $9.6 \%$ of patients aged $70+$ years $(p$ value $<0.001)$. Male patients were more likely to have CRC than female patients ( $7.1 \%$ vs $4.3 \%)$. Among patients who had a colonoscopy, $14.7 \%$ of patients with a GP label of HSCan were diagnosed with CRC compared to $4.7 \%$ of patients who had no GP label of HSCan ( $p$ value $<0.001)$, and $17.2 \%$ of patients with a hospital label of HSCan were diagnosed with CRC compared to $5.1 \%$ of patients who had no hospital label of HSCan $(p$ value $<0.001)$. The proportion of patients who had CRC was similar by ethnicity, year of referral, whether it was direct access colonoscopy, and whether the GP practice was a high referrer.

After adjustment for age, gender, ethnicity, year of referral, whether it was direct access colonoscopy or not, whether the GP practice was a high referrer or not, hospital label of HSCan and interaction term, the odds ratio of a GP label of HSCan in being diagnosed with CRC was 2.34 (95\% CI 1.70-3.22). The adjusted odds ratio of a hospital label of HSCan in being diagnosed with CRC was 2.43 (95\% CI 1.18-5.02). The odds ratio of age (for each additional year) and gender (men compared to women) in being diagnosed with CRC was 1.05 (95\% CI 1.041.06) and 1.67 (95\% CI 1.35-2.07), respectively (Table 4). There was no difference in the risk of an underlying CRC for Māori compared to non-Māori or for high referrers compared to low referrers. A subgroup analysis showed that for the 423 patients that were labelled as HSCan by their GP but not by the hospital triage process the underlying risk of CRC was $13.0 \%$ (Table 5).

\section{Discussion}

Colonoscopy is a common diagnostic procedure in patients referred to general surgery or gastroenterology, with $32.5 \%$ of patients undergoing the procedure. Thus in the current study, approximately $1.6 \%(6346 / 400,000)$ of patients residing in the Waikato DHB over a 3 year period underwent colonoscopy. This is similar to the $2.0 \%$ found in the Netherlands [11], although the proportion who were found to have CRC in the current sample was greater. While this study was conducted before the Covid pandemic reached NZ and before the Waikato DHB introduced free bowel screening for those age 60-74 years, we believe the referral rates for patients with bowel symptoms to our specialist services are unlikely to change substantially. We found older patients and those who had an HSCan label were more likely to receive a colonoscopy. This is unsurprising considering the risk of pathology increases with age. If the clinical picture suggests cancer then these patients should be prioritised. There was a small and probably clinically insignificant difference in the rate of cases accepted for colonoscopy after referral from high referring general practices. This may be due to different risk indicators in patients referred by high referrers. After adjustment for other factors, Māori were $34.0 \%$ less likely to have a colonoscopy. While Māori have a lower incidence of CRC than nonMāori, the size of the difference was surprising and needs further investigation. We know that there are differences in the treatment of Mãori patients in NZ with CRC [15] e.g., Mãori with CRC are less likely to receive surgery or adjuvant chemotherapy.

The study has shown that the conversion rate for CRC following colonoscopy in patients referred from GPs to specialist public hospital care is $5.5 \%$. This is similar to the conversion rate of $4.7 \%$ found in the national screening pilot [12] where patients underwent colonoscopy following a positive FIT. This does not mean that $94.5 \%$ are negative, as a significant proportion of patients will have adenoma or other relevant pathology-as was found in the screening program [12]. The use of FIT can help rule out CRC in patients presenting in primary care with symptoms [16], however, FIT is not routinely available as a diagnostic tool for NZ GPs. Thus, it is possible that even greater efficiency could be gained in the diagnostic pathway for symptomatic patients which would free up colonoscopy facilities for screening purposes. When considering the underlying likelihood of CRC being found, 
Table 3 Characteristics of patients who had a colonoscopy

\begin{tabular}{|c|c|c|c|c|c|c|}
\hline Characteristics & No CRC & & Had CRC & & $p$ value & Overall \\
\hline \multicolumn{7}{|l|}{ Age group } \\
\hline $30-49$ & 1225 & $98.5 \%$ & 19 & $1.5 \%$ & $<0.001$ & 1244 \\
\hline $50-59$ & 1335 & $96.7 \%$ & 46 & $3.3 \%$ & & 1381 \\
\hline $60-69$ & 1753 & $95.1 \%$ & 90 & $4.9 \%$ & & 1843 \\
\hline $70+$ & 2033 & $90.4 \%$ & 217 & $9.6 \%$ & & 2250 \\
\hline \multicolumn{7}{|l|}{ Gender } \\
\hline Female & 3627 & $95.7 \%$ & 163 & $4.3 \%$ & $<0.001$ & 3790 \\
\hline Male & 2719 & $92.9 \%$ & 209 & $7.1 \%$ & & 2928 \\
\hline \multicolumn{7}{|l|}{ Ethnicity } \\
\hline Non-Māori & 5710 & $94.5 \%$ & 334 & $5.5 \%$ & 0.904 & 6044 \\
\hline Māori & 636 & $94.4 \%$ & 38 & $5.6 \%$ & & 674 \\
\hline \multicolumn{7}{|l|}{ Year } \\
\hline 2015 & 2207 & $95.3 \%$ & 108 & $4.7 \%$ & 0.056 & 2315 \\
\hline 2016 & 2095 & $93.7 \%$ & 140 & $6.3 \%$ & & 2235 \\
\hline 2017 & 2044 & $94.3 \%$ & 124 & $5.7 \%$ & & 2168 \\
\hline \multicolumn{7}{|c|}{ Direct colonoscopy } \\
\hline No & 2261 & $93.9 \%$ & 148 & $6.1 \%$ & 0.104 & 2409 \\
\hline Yes & 4085 & $94.8 \%$ & 224 & $5.2 \%$ & & 4309 \\
\hline \multicolumn{7}{|l|}{ High referrer } \\
\hline Low & 2202 & $94.9 \%$ & 119 & $5.1 \%$ & 0.285 & 2321 \\
\hline High & 4144 & $94.2 \%$ & 253 & $5.8 \%$ & & 4397 \\
\hline \multicolumn{7}{|l|}{ HSCan-GP } \\
\hline No & 5847 & $95.3 \%$ & 286 & $4.7 \%$ & $<0.001$ & 6133 \\
\hline Yes & 499 & $85.3 \%$ & 86 & $14.7 \%$ & & 585 \\
\hline \multicolumn{7}{|l|}{ HSCan-hospital } \\
\hline No & 6154 & $94.9 \%$ & 332 & $5.1 \%$ & $<0.001$ & 6486 \\
\hline Yes & 192 & $82.8 \%$ & 40 & $17.2 \%$ & & 232 \\
\hline Overall & 6346 & $94.5 \%$ & 372 & $5.5 \%$ & & 6718 \\
\hline
\end{tabular}

age was obviously a significant factor, with a steep rise in risk with age from $1.5 \%$ in younger patients to $9.6 \%$ of patients $70+$ years having CRC. Men were much more likely to have $\mathrm{CRC}$ with a $7.1 \%$ conversion rate compared with women at $4.3 \%$. These findings support the guidance for referral. However, we know that there is also an increase in the incidence of CRC in younger patients in NZ [17] and if cases are not to be missed it may still be worthwhile offering colonoscopy to younger patients in order to exclude cancer. While there was no difference in the likelihood of Māori undergoing colonoscopy having CRC (5.6\% vs 5.5\% in non-Māori) we know the incidence of CRC in Māori is less than in non-Māori. If Māori rates of colonoscopy were similar to non-Māori we may find that the positivity rate would fall in line with the known lower incidence of CRC in Māori. The characteristics of the general practice where patients were registered did not seem to influence the conversion rate-thus those patients referred for direct colonoscopy did not differ, and there was no difference in the rate of CRC for high referrers compared to low referrers. However, if the GP had indicated an HSCan and a colonoscopy was carried out, then the conversion rate was $14.7 \%$. While the rate in those deemed an HSCan by the hospital specialist team was higher at $17.2 \%$, this was based on only 232 cases. Seventy two percent $(423 / 585)$ of GP HSCan patients were downgraded resulting in 55/423 (13.0\%) patients deemed as having an HSCan by their GP having an unnecessary delay in diagnosis due to the hospital triage process. We could argue that the sensitivity and specificity of a GP identification of an HSCan is such that all these patients should be offered an urgent colonoscopy. The poor outcomes in NZ from CRC have been linked to late diagnosis and any opportunity to expedite a diagnosis rapidly could be considered worthwhile.

\section{Strengths and limitations}

A study strength is that we have outcome data on over 6000 colonoscopy cases following referral from GPs. We did not have data on patients who were invited to have a 
Table 4 Adjusted odds ratio of having colorectal cancer

\begin{tabular}{|c|c|c|c|}
\hline Factors & $P$ value & Odds ratio & $\begin{array}{l}95 \% \\
\text { confidence } \\
\text { interval }\end{array}$ \\
\hline \multicolumn{4}{|l|}{ Age } \\
\hline (continuous) & $<0.001$ & 1.05 & $(1.04-1.06)$ \\
\hline \multicolumn{4}{|l|}{ Gender } \\
\hline Female & Ref & & \\
\hline Male & $<0.001$ & 1.67 & $(1.35-2.07)$ \\
\hline \multicolumn{4}{|l|}{ Year } \\
\hline (continuous) & 0.606 & 1.04 & $(0.90-1.19)$ \\
\hline \multicolumn{4}{|l|}{ Ethnicity } \\
\hline Non-Māori & Ref & & \\
\hline Māori & 0.067 & 1.40 & $(0.98-2.01)$ \\
\hline \multicolumn{4}{|l|}{ High referrer } \\
\hline Low & Ref & & \\
\hline High & 0.814 & 1.03 & $(0.82-1.29)$ \\
\hline \multicolumn{4}{|l|}{ Colonoscopy } \\
\hline FSA and colonoscopy & Ref & & \\
\hline Direct colonoscopy & 0.564 & 0.94 & $(0.75-1.17)$ \\
\hline \multicolumn{4}{|l|}{ HSCan-GP } \\
\hline No & Ref & & \\
\hline Yes & $<0.001$ & 2.34 & $(1.70-3.22)$ \\
\hline \multicolumn{4}{|l|}{ HSCan-hospital } \\
\hline No & Ref & & \\
\hline Yes & 0.016 & 2.43 & $(1.18-5.02)$ \\
\hline \multicolumn{4}{|l|}{ Interaction term } \\
\hline (HSCan-GP $\times$ HSCan-Hospital) & 0.342 & 0.65 & $(0.27-1.57)$ \\
\hline
\end{tabular}

Table 5 Proportion of patients diagnosed with CRC following colonoscopy by HSCan category

\begin{tabular}{llll}
\hline & GP HSCan & No GP HSCan & Totals \\
\hline Hospital HSCan & $31 / 162(19.1 \%)$ & $9 / 70(12.9 \%)$ & $40 / 232(17.2 \%)$ \\
No hospital HSCan & $55 / 423(13.0 \%)$ & $277 / 6063(4.6 \%)$ & $332 / 6486(5.1 \%)$ \\
\hline
\end{tabular}

colonoscopy but did not attend. Study data included both patient and GP characteristics. A weakness is that we did not have complete data on symptoms, or the reason for referral. In addition, outcome data only included a diagnosis of CRC derived from the Cancer Registry. Therefore, we did not have information on other pathology found at colonoscopy.

\section{Implications}

The implications of these findings for policy include the need for NZ Bowel Cancer Guidelines to reassess the use of the HSCan and 2 week wait rule for patients deemed at high suspicion of cancer by their GP. We would argue that all patients deemed at high risk by their GP should be offered timely colonoscopy and that further delay by an additional triage step by the hospital in the referral pathway is unnecessary. We also believe that it is timely for $\mathrm{NZ}$ to review their guidelines for diagnosis in the light of the UK NICE guidance [18] and introduce the option of a FIT test in general practice to help rule out the need for referral for colonoscopy. Finally, given the poor outcomes for Māori following a diagnosis of CRC, the finding of a lower use of colonoscopy is of concern.

\section{Conclusions}

Almost six percent of colonoscopies in symptomatic patients referred by general practitioners result in a finding of CRC. The likelihood of cancer increases with age and is greater in men. If the GP identifies a high risk of cancer then the likelihood of a positive colonoscopy is almost $15 \%$, suggesting that these patients should be routinely prioritised without the need for further hospital triage. Further research is needed to understand why Mãori are less likely to receive a colonoscopy following referral from general practice.

\section{Abbreviations \\ NZ: New Zealand; CRC: Colorectal cancer; GP: General practitioner; DHB: Dis- trict Health Board; $\mathrm{MOH}$ : Ministry of Health; HSCAn: High suspicion of cancer; UK: United Kingdom; FSA: First Specialist Assessment; NHI: National Health Index; OR: Odds ratio; Cl: Confidence interval; FIT: Faecal Immunochemical test.}

\section{Acknowledgements}

We would like to thank the Waikato DHB and the National Cancer Register for provision of the data.

\section{Authors' contributions}

RL wrote the main manuscript. CL and SM conducted the data analysis. TB prepared the manuscript for publication. All authors read and approved the final manuscript.

\section{Funding}

This work was supported by the Health Research Council of New Zealand (HRC Grant Number 17/147).

\section{Availability of data and materials}

The data analysed for the current study are not publicly available for ethical reasons. All data relevant to the study are included in the article. Anonymised data can be made available from the corresponding author on request.

\section{Declarations}

\section{Ethics approval and consent to participate}

All methods were performed in accordance with The Declaration of Helsinki and ethical approval was granted by the New Zealand Health and Disability Ethics Committee (Ethics ref: 17/NTB/157). Patients were not involved in the design, or conduct, or reporting, or dissemination plans of the research. This is a retrospective analysis of electronic records; as such data were analysed anonymously and no patient identifiers have been published. Therefore, informed consent was waived by the New Zealand Health and Disability Ethics Committee (Ethics ref: 17/NTB/157). 


\section{Consent for publication \\ Not applicable.}

\section{Competing interests}

The authors declare that they have no competing interests.

\section{Author details}

${ }^{1}$ Medical Research Centre, University of Waikato, Hamilton, New Zealand. ${ }^{2}$ Waikato District Health Board, Hamilton, New Zealand. ${ }^{3}$ Auckland University of Technology, Auckland, New Zealand. ${ }^{4}$ Department of General Practice and Rural Health, University of Otago, Dunedin, New Zealand. ${ }^{5}$ School of Population Health, University of Auckland, Auckland, New Zealand. ${ }^{6}$ Centre for Population Health Sciences, The University of Edinburgh, Edinburgh, Scotland, UK. ${ }^{7}$ Medicine, Dentistry and Health Sciences, The University of Melbourne, Melbourne, Australia.

Received: 6 September 2021 Accepted: 25 November 2021

Published online: 15 December 2021

\section{References}

1. Ministry of Health. Cancer: new registrations and deaths 2017. Wellington: Ministry of Health; 2019. Accessed 15 May 2020.

2. Ministry of Health. Cancer: new registrations and deaths 2013. Wellington: Ministry of Health; 2016. Accessed 15 May 2020.

3. Williams TG, Cubiella J, Griffin SJ, Walter FM, Usher-Smith JA. Risk prediction models for colorectal cancer in people with symptoms: a systematic review. BMC Gastroenterol. 2016;16(1):63.

4. Cunningham D, Atkin W, Lenz H-J, Lynch HT, Minsky B, Nordlinger B. Colorectal cancer. Lancet. 2010;375(9719):1030-47.

5. Ministry of Health. Bowel cancer quality improvement report. Wellington: Ministry of Health; 2019. Accessed 21 May 2020.

6. Sharples K, Firth M, Hinder V, Hill A, Jeffery M, Sarfati D, et al. The New Zealand PIPER Project: colorectal cancer survival according to rurality, ethnicity and socioeconomic deprivation-results from a retrospective cohort study. NZ Med J. 2018;131(1476):24-39.

7. New Zealand Guidelines Group. Suspected cancer guideline ebook. Wellington: NZ: New Zealand Guidelines Group; 2012. Accessed 20 Apri 2020.

8. Lawrenson R, Logie J, Marks C. Risk of colorectal cancer in general practice patients presenting with rectal bleeding, change in bowel habit or anaemia. Eur J Cancer Care. 2006;15(3):267-71.

9. Ministry of Health. Referral criteria for direct access outpatient colonoscopy or computed tomography colonography. Wellington: NZ: Ministry of Health; 2019. Accessed 15 May 2020.

10. Hamilton W, Lancashire R, Sharp D, Peters TJ, Cheng K, Marshall T. The risk of colorectal cancer with symptoms at different ages and between the sexes: a case-control study. BMC Med. 2009;7(1):17.

11. Koning NR, Moons LM, Büchner FL, Helsper CW, ten Teije A, Numans ME. Identification of patients at risk for colorectal cancer in primary care: an explorative study with routine healthcare data. Eur J Gastroenterol Hepatol. 2015;27(12):1443-8.

12. Smith $L$, Borman $B$, Love $T$. Final evaluation report of the bowel screening pilot: screening rounds one and two. Wellington: NZ: Ministry of Health: 2016

13. Mjoli M, Govindasamy V, Madiba TE. What is the diagnostic yield of colonoscopy in patients with a referral diagnosis of constipation in South Africa? S Afr J Surg. 2017:55(3):14-8.

14. Murchie P, Chowdhury A, Smith S, Campbell NC, Lee AJ, Linden D, et al. General practice performance in referral for suspected cancer: influence of number of cases and case-mix on publicly reported data. Br J Cancer. 2015;112(11):1791-8.

15. Hill S, Sarfati D, Blakely T, Robson B, Purdie G, Dennett E, et al. Ethnicity and management of colon cancer in New Zealand: do indigenous patients get a worse deal? Cancer. 2010;116(13):3205-14.

16. Bailey SE, Abel GA, Atkins A, Byford R, Davies S-J, Mays J, et al. Diagnostic performance of a faecal immunochemical test for patients with low-risk symptoms of colorectal cancer in primary care: an evaluation in the South West of England. Br J Cancer. 2021;124(7):1231-6.
17. Gandhi J, Davidson C, Hall C, Pearson J, Eglinton T, Wakeman C, et al. Population-based study demonstrating an increase in colorectal cancer in young patients. Br J Surg. 2017;104(8):1063-8.

18. National Institute for Health and Care Excellence. Quantitative faecal immunochemical tests to guide referral for colorectal cancer in primary care. Diagnostics guidance. UK: NICE; 2017

\section{Publisher's Note}

Springer Nature remains neutral with regard to jurisdictional claims in published maps and institutional affiliations.
Ready to submit your research? Choose BMC and benefit from:

- fast, convenient online submission

- thorough peer review by experienced researchers in your field

- rapid publication on acceptance

- support for research data, including large and complex data types

- gold Open Access which fosters wider collaboration and increased citations

- maximum visibility for your research: over $100 \mathrm{M}$ website views per year

At BMC, research is always in progress.

Learn more biomedcentral.com/submissions 